\title{
NEWS
}

\section{Nanoscale food protection}

\author{
John A. Glaser ${ }^{1}$
}

Published online: 12 April 2015

(C) Springer-Verlag Berlin Heidelberg (Outside the USA) 2015

Consumption of food contaminated with pathogenic microorganisms or their toxins leads to a host of diseases resulting in serious economic and public health consequences. Some 48 million people in the US annually succumb to foodborne diseases with the hospitalization of 128,000 and death to 3000 people. Pathogenic microorganisms can contaminate fruits and vegetables during the passage through their extensive production chain from field production, intermediate handling, distribution, to food industry, and home utilization. Current disinfection techniques include: chlorine/hypochlorite, chlorine dioxide, peracetic acid, hydrogen peroxide, quaternary ammonium salts, ozone, and UV irradiation. A new technology exhibits inactivation of microorganisms on fresh produce, and food production surfaces to be controlled by water nanostructures generated by electrospraying water vapor. The intervention method utilizes engineered water nanostructures (EWNS) formed in an electrospray with unique properties as a diameter of $25 \mathrm{~nm}$, involving reactive oxygen species, a strong surface charge of $10 \mathrm{e} /$ structure, and can be airborne for hours indoors. The electrospray module consisted of an electrostatic precipitator commonly used to aerosolize particles and functionally designed to deliver EWNS to surfaces.

The new technology's efficacy for inactivating representative foodborne bacteria like Escherichia coli, Salmonella enterica, and Listeria innocua on stainless steel surfaces and on organic tomatoes was evaluated. A $1.4 \log$ reduction for

John A. Glaser

glaser.john@epa.gov

1 National Risk Management Research Laboratory, US Environmental Protection Agency, 26 W King Dr, Cincinnati, OH 45268, USA
E. coli on organic tomato surfaces was found using the EWNS technology for an aerosol concentration of $50,000 \mathrm{cfu} / \mathrm{cm}^{3}$ at 90 min of exposure compared with control using the EWNS (same temperature and relative humidity). For L. innocua, the dose-response relationship was demonstrated. On surfaces, bacterial concentrations were reduced depending on the microorganism and surface type. The inventors of this technology look for this novel, chemical-free, and environmentally friendly intervention method to offer potential for development and application in the food industry, as a "green" alternative to existing disinfection methods.

Environ. Sci. Technol., 2015, 49 (6), pp. 3737-3745.

\section{Disbelief in science for the current age}

Remarkably, the journal National Geographic has undertaken a review/survey of the currently held views of science and technology held throughout society. In this age, scientific information ranging from the safety of vaccines and fluoride to climate change are objects of organized opposition and derision. Opponents are fueled with their own literature and sources of information from which they assault scientific and technical information. The doubters are enabled through selected sources of information and their own interpretations of research to declare war on the consensus of experts. At this time, controversies range across a remarkable landscape of disciplines and information that "science doubt" appears to have become a pop-culture theme. The pervasive presence of science and technology in modern society may actually draw out a counter position.

Society confronts threats and risks associated with new inventions in a variety of ways. If we consider genetically modified organisms, some wonder about the veracity of experts' testimony asserting that there is no evidence that 
these genetic constructs are detrimental to human health and safety. The techniques employed to precisely alter genes in the laboratory are comparable to traditional breeding in terms of additional risk or danger. Yet one of society's responses is to portray these techniques to be akin to those of the protagonist in Mary Shelley's Frankenstein. The food derived from these actions using mad science was contemptuously named Frankenfood. The recent societal reaction to the Ebola virus displayed a remarkable degree of the doubter mentality. The prominence of personal risk reigned high among the uninformed to the point that the doubter crowd was willing to consider mutation of the virus to present an airborne threat and hence a superplague.

Distinguishing real from imaginary hazards occupies the attention of the public where confusion can reign amidst the doubter contribution of information denying the validity of scientific and technical findings pertinent to decision making about these hazards. Are we to be held hostage by the doubter philosophy which contributes confusion to matters such as the Ebola virus and the claim that there is a possibility that the virus can mutate to an airborne superplague? These dystopic ruminations serve to heighten public concern without any real basis of fact. Zero evidence is available to show that the complete change of the virus to a different mode of transmission is possible. Scientific consensus points out that such a consequence is extremely unlikely. Other appalling instances of doubter philosophy in operation can be found in the news relative to evolution, vaccines, climate change, and fluoridation of drinking water. Lest we become dismayed with the current situation, a quick reflection to past history offers an impressive list of doubter philosophy applications to matters such as the relative positions of the earth and sun within stellar space.

Our reaction to the various claims concerning decision making and risk is something that we relearn multiple times owing to the apparent forgetfulness of the basic principles involved. The selection of what to believe is bewildering when we are faced with decisions related to risk and the action required. "Science is not a body of facts," emphasizes Marcia McNutt, current Science editor. She adds that "Science is a method for deciding whether what we choose to believe has a basis in the laws of nature or not."

Nat Geograph 2015, 227, 3, 30-47.

\section{Membrane distillation}

Membrane distillation (MD) is a separation technique based on vapor transport across a hydrophobic microporous membrane. Vapor transport is determined by the vapor pressure gradient across the membrane. Separation processes utilizing mass flows by a membrane, generally employ static pressure differences to drive the separation. Such is the driving force between the two bounding surfaces as in the case of reverse osmosis, a difference in concentration differences in dialysis or through the application of an electric field. Membrane selectivity is a function of pore size relative to the retained substance, its diffusion coefficient, or electrical polarity. Membrane distillation requires a membrane selectivity based on the retention of liquid water with concurrent permeability for free water vapor. The membranes are constructed of hydrophobic synthetic materials such as PTFE, PVDF, or PP having standard diameter pores between 0.1 and $0.5 \mu \mathrm{m}$. The membrane composition is non-polar hence the membrane material is not wetted by dipolar liquids such as water. The high water surface tension controls penetration even through larger pores. Separation proceeds through means of phase change while driven through thermal changes. A partial vapor pressure difference initiated by a temperature difference is the driving force for the process. Seawater desalination, wastewater treatment, separation of volatile compounds, concentration of non-volatile compounds from various mixtures, and dairy fluid processing are potential areas for the application of membrane distillation.

Desalination 2015, 356, 56-84.

\section{Green chemical process and synthesis design using life-cycle assessment}

A tutorial review explores how life-cycle assessment and related methods can be applied to green chemical process development and synthesis design. This analysis of the development of greener process and synthesis concepts offers a deeper understanding of compound selection and process methodology dependency leading to reduced environmental impacts. Emerging research areas of nanotechnology, flow chemistry, process intensification, process integration, waste reduction and novel treatment, alternative energy source uses, renewable resources harvested with new chemistries and solvents offer opportunities for chemical process redesign.

The utility of LCA approaches to chemical process and product design within a spectrum of research and development applications offers the opportunity to scrutinize case studies as applied to the assessment of emerging technologies and procedures, optimization of conventional processes, and decision-making support for public and private entities related to process changes. Comparisons of technology enable the fruitful inspection of traditional 
chemical processing technology with a series of more sustainable green chemistry-based technologies.

Different life-cycle approaches including gate-to-gate and cradle-to-grave scenarios with impact assessment methods were applied to the case studies depending on the specific analytical questions. Standardized guidelines relating the specific needs of a research field are required to ensure the quality of the LCA methodology application.

The investigation of biphasic esterification of phenol with benzoyl chloride to form phenyl benzoate under moderate reaction conditions utilized a combination of Simplified LCA and cost analysis in the following scheme.

\section{US renewable energy advances}

In the 277 page February 5, 2015 monthly installment of the Electric Power Monthly, the U.S. Energy Information Administration provides statistics covering data through December 2014 concerning the emerging role of light solar and wind energy to electrical power generation. Solar photovoltaic (PV) systems generated $15,874 \mathrm{GWh}$ of power last year, with an increase over the $121 \mathrm{GWh}$ of 2013. Solar thermal generation accelerated faster to $2447 \mathrm{GWh}$ in 2014 from $915 \mathrm{GWh}$ in 2013. Smaller than $1 \mathrm{MW}$ generating systems, a major

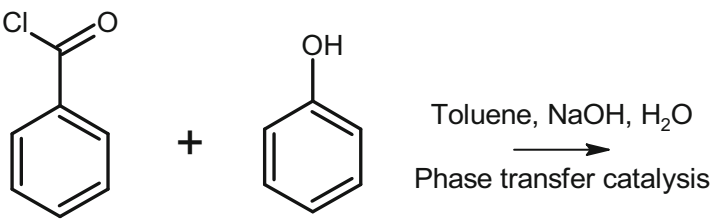<smiles>O=C(Oc1ccccc1)c1ccccc1</smiles>

The biphasic reaction system mixing was improved by changing the synthesis conditions to flow processes from batch conditions. Process parameters significantly affected the yield such as the selection of ionic liquids such as $\left[\mathrm{C}_{18} \mathrm{MIM}\right] \mathrm{Br}$, [MIM] $\left[\mathrm{BuSO}_{3}\right]$, or $[\mathrm{BMIM}] \mathrm{Br}$ as phase transfer catalysts. The esterification reaction was remarkable enhanced by phase transfer catalysis when contrasted with non-catalyzed reaction conditions. The ionic liquid catalysts were found to improve the environmental balance despite the high environmental burden incurred through their use. Sensitivity analysis was conducted to determine optimal relevant process parameters. Despite the high environmental burden of these compounds resulting from their material and energy demanding synthesis, the overall environmental balance was improved even when the ionic liquids were used only once without recycling. For this reaction sensitivity analyses were performed by varying relevant process parameters to select optimal synthetic conditions. The reaction work-up was found to be a major obstacle and was optimized for product flow and energy use. In the flow system, the high electricity demand of the peripheral equipment was reduced through the production optimization. This accomplishment underscores the general utility of Simplified LCA and in this case a cost analysis to improve process conditions and economics.

Green Chemistry 2015, 17, 123-145; 2013, 13, 1694-1707. contributor to new distributed and rooftop systems, could significantly alter the overall solar share. Research data show that residential and commercial PV systems were strong contributors to the new solar power being installed through 2013. Wind power generated $181,179 \mathrm{GWh}$ in 2014 , an increase of about $14,000 \mathrm{GWh}$ over 2013 generation numbers. The rate of solar power adoption currently exceeds that of wind power and many other forms of renewable energy.

Solar PV's share of electricity supply still lags far behind wind power. However, solar is growing much faster than wind power. Natural gas-fired electricity generation slumped in 2013 but this trend may be easily reversed due to the plants planned for construction during 2015 coming on-line. At the same time, new solar and wind power projects planned to come on-line this year far outnumber new gas-fired power plants are clearly outnumbered by new solar and wind construction projects.

http://www.eia.gov/electricity/monthly/pdf/epm.pdf.

\section{Graphene oxide simple sonosynthesis}

A graphene sheet is composed of two-dimensional layers of $s p^{2}$ hybridized carbon of one-atom thickness. This material can have huge theoretical specific surface areas of $2630 \mathrm{~m}^{2} / \mathrm{g}$ with high intrinsic mobility, high Young's modulus, thermal conductivity, and remarkable optical transmittance. Such properties herald graphene and 
graphene oxide as promising source materials for construction of multifunctional composites with applications as high performance electrodes, and catalysts. edges of graphene planes. Graphene-based sensors for glucose detection actually perform better than corresponding sensors without graphene.
Expanded graphite (10 or 30 um)

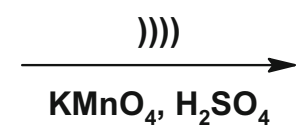

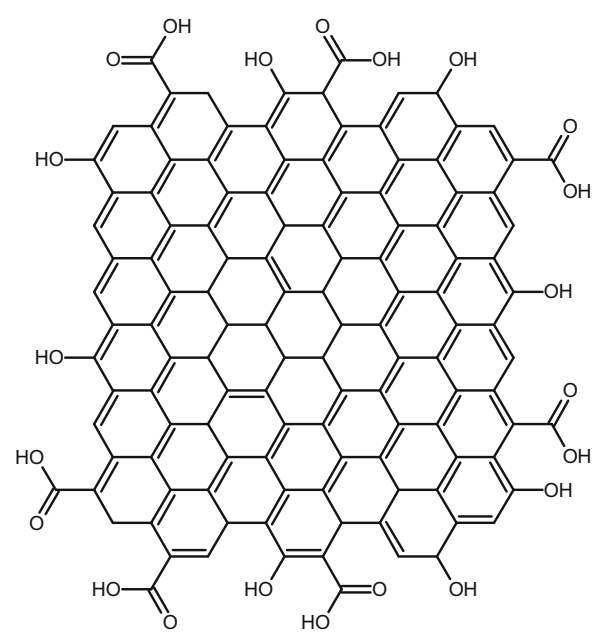

Graphene oxide
A simple synthesis of graphene oxide employs expanded graphite in an ultrasonic cleaner. The new method involves a synergistic effect between intercalation (oxidation) and ultrasonication with reduced acid demand, higher productivity, reduced energy demand, avoidance of toxic gas release, and shorter reaction times in contrast with earlier synthetic approaches. Graphene oxide (GO) sheets having layer spacings of $\sim 1 \mathrm{~nm}$ were formed by this methodology in less than $1 \mathrm{~h}$. This procedure was safe and expected to be applicable for the scale production of GO.

While there are simplified means to access graphene and graphene oxide current analytical methods are insufficient to address the details of chemical modifications provided by synthetic chemists. An increasing library of covalent and non-covalent methodologies is directed to chemical functionalization of graphene and graphene oxide. The reaction products will require sophisticated analytical methods. The development of fundamental structural information and chemical properties of graphene and graphene oxide continue as a prerequisite for the successful development synthetic modifications and practical applications of the new synthetic products. One significant application is the development of glucose detection sensors necessary for diabetic blood monitoring. The preparation, properties, and efficiency of the detection processes are important to the successful development of these sensors. For instance, graphene may display different types of defects important to electron transfer processes, and conductivity is reduced by oxygenated groups situated at the
Ind Eng Chem Res 2014, 53, 17878-17883; Angew Chem Int Ed 2014, 53, 7720-7738; Electroanalysis 2015, 27, 16-31.

\section{Diazo continuous process chemistry}

Synthetic organic chemistry relies on a variety of materials to assemble structurally complex molecules. As highly versatile reagents, diazo compounds are found in many small scale synthetic steps but have explosive features that make them extremely difficult to handle on an industrial scale. Diazo compounds are clean and atom-efficient reagents making them very important to the synthetic considerations of green chemistry with their leaving group as dinitrogen. Diazo compounds release one equivalent of nitrogen upon decomposition/reaction leading to significant pressure buildup with large-scale batch conditions. These limitations translate into rare industrial, large-scale use. Often less efficient, waste generating and increased energy consuming synthetic routes are employed for safety considerations.

Microstructured continuous flow technology can enable the use of these attractive reagents at large scale. The explosive features are directly connected to the nitrogen leaving group that is hallmark to these reagents. Micro devices can enable their use through heat transfer improvements and increased operational safety by reduction of known conditions of explosive concern. Utilization of 
these reagents at small scale and in small quantities through in situ formation of the reagents enables the design of continuous process equipment to meet the quantity generation needs on a timely basis. $\beta$-ketoesters can be synthesized from aldehydes and ethyl diazoacetate as depicted in the following scheme.
OECD document strongly asserts that favoring fossil fuel over clean energy investments should be avoided by any government policy. The report selects a "step change" to clean energy investment as a necessity, should the world's nations desire control of global warming to less than $2{ }^{\circ} \mathrm{C}$ for this century. This United Nations threshold has been selected<smiles>CCOC(=O)CNCl</smiles>

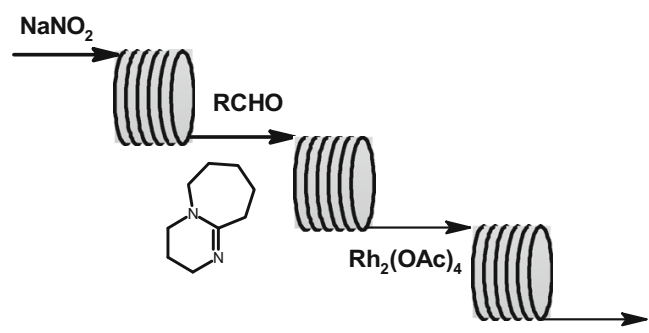<smiles>[R]C(=O)CC(=O)OCC</smiles>

Ethyl diazoacetate is formed in water in the first reactor followed by addition to aldehydes in DMSO within a second reactor yielding $\alpha$-diazo- $\beta$-hydroxyesters in high yields with short residence times and high concentrations. A third reactor accomplished conversion to $\beta$-ketoesters through a rhodium acetate catalyzed 1,2-hydride shift. The succinct path of three easily managed steps enhances the process as a prospective industrial application. Diazo compounds can clearly be desirable reagents supporting sustainability through their ability to reduce the number of synthetic steps and waste generated and now handled as safe and easy to manage steps.

The desirable atom-economic reactions required to meet the synthetic challenges related with valuable products, such as pharmaceuticals, materials, and fertilizers, must be environmentally benign and safe. The unique properties and reactivities of diazo reagents in terms are their atomeconomy produce target chemicals with high chemo-, regio-, and stereoselectivity. Microreactor-based continuousflow technology provides the start to a nascent platform to enable chemists to utilize the features of diazo compounds as major contributor to process chemistry of the future.

ChemSusChem 2015, 8, 245-250.

\section{Clean energy growth needs legal protection}

In a recently released 116 page policy report, the Organization for Economic Cooperation and Development (OECD) calls for governments to ensure protection to foreign investors in clean energy infrastructure comparable to protection afforded domestic and state-owned companies. The to reflect major problems linked to climate change. Strained public finances invite the development of financing from private sources and the development of a supporting infrastructure for clean energy, including electricity generated from solar, wind, hydro, geothermal, marine, biomass, and waste-to-energy; biofuels; energy-smart technologies, such as smart grids; energy efficiency technology; and electric vehicles. The report underscores market and government "failures," as fossil fuel subsidies, lack of supportive policies, and persistent barriers to international trade and financing, that constrain clean energy investment. Renewable energy investment was found to be significantly impeded by weak environmental policy. Clean energy goals must be "robust and credible" and coupled with the elimination of fossil fuel subsidies and carbon emission costing. Since the energy sector is generally found to be more restrictive than other sectors for foreign direct investment, governments should assess the economic and environmental impacts of foreign ownership restrictions in clean energy.

http://www.oecd-ilibrary.org/docserver/download/2014 061e.pdf? expires $=1425572212 \&$ id $=$ id $\&$ accname $=$ guest $\&$ checksum=D3178734E3075141F5C456AA66AE033E.

\section{Pharma industry use of single-use technologies}

The requirements of the pharmaceutical industry to produce certain therapeutic items require manufacturing conditions that are at the acme of "clean technology." During 
the past 10 years, single-use technologies in the biopharmaceutical industry have dominated small- and mid-scale bioprocessing with significant instances of these very helpful devices scaled to larger quantity manufacturing. Comparison of single-use versus fixed stainless steel systems has been conducted within the pharmaceutical industry. Rapid deployment of bioprocessing across multiple scales within the same manufacturing area has shown the power of single-use equipment. The unknowns of a decade ago concerning single-use equipment are no longer of any importance and in general construction composition is much improved to include advanced design, multilayer laminated plastic bags, and other integrated technologies. Trends and forecasts of future developments continue to offer an increasingly attractive technology.

A special application using single-use and modular systems for vaccine production is offered in detail. The current manufacturing technologies for most vaccines were developed 40-50 years ago with facilities of similar age resulting in the manufacture of costly, complex, uncharacterized products. The vaccine industry today struggles to provide improved existing products and new vaccines economically. Each vaccine manufacturing process is quite unique and is not pliable to the development of standard platform processes and the design of supporting facilities comparable to antibody manufacturing. A new paradigm has been developed to exploit single-use technologies, specific engineering, and design directions to formulate vaccine manufacturing facilities based on modular construction. This new approach offers the opportunity for rapid construction of vaccine facilities at a significant reduction in capital expenditure. This new conceptualization of a vaccine manufacturing plant was simulated through use of an inactivated polio vaccine as a model. The new plant has the characteristics of rapid deployment at different locations responsive to market and/or tender constraints without risk or cost associated with process architecture and drug product changes.

Engin Life Sci 2014, 14, 238-243; 244-253; Gen Eng Biotech News 2014, 34 (14), 24-27.

\section{New journal}

\section{Frontiers in environmental science}

This journal seeks to utilize a unique platform for openaccess publishing connected with research network exchange to provide enhanced opportunities for communication and creation of knowledge. Frontiers in Environmental Science has been designed to publish articles relating discoveries across the research spectrum connected to the field of environmental science. Specialty sections of this new journal are mini reviews, perspectives, original research, opinion, editorials, and specialty grand challenge and technology reports. The journal espoused an operating philosophy whereby working scientists are prominent to an interactive, fair, and efficient review process. Objective editorial criteria are derived from established guidelines and assisted by a scientific/technical scrutiny within the peer-review process. Specialty subject sections of this journal include: agroecology and land use systems, air pollution, atmospheric science, environmental health, environmental informatics, environmental toxicology, green and environmental chemistry, groundwater resources and management, interdisciplinary climate studies, marine pollution, microbiotechnology, ecotoxicology, bioremediation, soil processes, and wastewater management.

http://www.frontiersin.org/environmental_science. 